\title{
Repression of chsB expression reveals the functional importance of class IV chitin synthase gene chsD in hyphal growth and conidiation of Aspergillus nidulans
}

\author{
Masayuki Ichinomiya, $\uparrow$ Takayuki Motoyama,† Makoto Fujiwara, $\neq$ \\ Masamichi Takagi, Hiroyuki Horiuchi and Akinori Ohta
}

Department of Biotechnology, The

University of Tokyo, 1-1-1 Yayoi, Bunkyo-ku, Tokyo 113-8657, Japan
Author for correspondence: Hiroyuki Horiuchi. Tel: +8135841 5170. Fax: +8135841 8015. e-mail: ahhoriu@mail.ecc.u-tokyo.ac.jp

The functions of two previously identified chitin synthase genes in Aspergillus nidulans, chsB and chsD, were analysed. First, a conditional chsB mutant was constructed in which the expression of chsB is under the control of a repressible promoter, the alcA promoter, of $A$. nidulans. Under repressing conditions, the mutant grew slowly and produced highly branched hyphae, supporting the idea that chsB is involved in normal hyphal growth. The involvement of chsB in conidiation was also demonstrated. Next, double mutants of chsB and chsD were constructed, in which chsB was placed under the control of the alcA promoter and ChsD was replaced with the argB gene of $A$. nidulans. These double mutants grew more slowly than the chsB single mutant under high-osmolarity conditions. The hyphae of the double mutant appeared to be more disorganized than those of the chsB single mutant. It was also found that ChsD was functionally implicated in conidiation when the expression of chsB was limited. These results indicate the importance of the ChsD function in the absence of chsB expression. The roles of ChsB and ChsD in hyphal growth and in conidiation were supported by the analysis of the spatial expression patterns of chsB and chsD, using lacZ of Escherichia coli as a reporter gene.

Keywords: cell wall, conidiation, filamentous fungi

\section{INTRODUCTION}

Chitin is a major component of the cell walls of most filamentous fungi and a minor component of the cell walls of many yeasts. Chitin synthase genes have been isolated from many filamentous fungi and yeasts, and have been classified into at least five classes, I-V, according to sequence similarity (Bowen et al., 1992; Beth Din et al., 1996; Specht et al., 1996; Motoyama et

†These authors contributed equally to this work.

\#Present address: The Institute of Physical and Chemical Research (RIKEN), Wako-shi, Saitama 351-0198, Japan.

Abbreviations: AP, alkaline phosphatase; RACE, rapid amplification of cDNA ends.

The GenBank accession number for the the new version of the chsD nucleotide sequence is D83246. al., 1996; Aufauvre-Brown et al., 1997; XoconostleCázares et al., 1997). In Aspergillus nidulans, four chitin synthase genes, chsC, chs $A$, chsB and $c h s D$, which belong to class I, class II, class III and class IV, respectively, and a gene, $\operatorname{csm} A$, which encodes chitin synthase with a myosin motor-like domain, have been cloned in our laboratory (Yanai et al., 1994; Motoyama et al., 1994a, 1996; Fujiwara et al., 1997) Our chsD is also referred to as 'chsE' by Specht et al. (1996). The chitin synthase domain of CsmA, which belongs to class $\mathrm{V}$, is similar to the product of 'chsD' as reported by Specht et al. (1996).

The functions of chitin synthases in filamentous fungi have been analysed by gene disruptions or gene deletions. The respective null mutants of class I (Motoyama et al., 1994a), class II (Beth Din \& Yarden, 1994; Yanai et al., 1994) and class IV (Beth Din et al., 1996; Motoyama et al., 1996; Specht et al., 1996) chitin 
synthase genes of filamentous fungi exhibited no obvious phenotypic defect under standard conditions with the exception of that studied by Beth Din \& Yarden (2000). Recently, it was reported that a class II chitin synthase gene (CHS1) of the dimorphic yeast Candida albicans was essential even for the growth of the yeast form (Sudoh et al., 2000; Munro et al., 2001). Disruptions or deletions of class III (Yarden \& Yanofsky, 1991; Yanai et al., 1994; Borgia et al., 1996; Mellado et al., 1996) or class V (Specht et al., 1996; Aufauvre-Brown et al., 1997; Horiuchi et al., 1999) chitin synthase genes, with the exception of two class III genes (Gold \& Kronstad, 1994; Mellado et al., 1996), caused some phenotypic changes. These observations indicate that the respective chitin synthase genes (not all genes) alone do not bear essential functions for hyphal growth and conidiation, and that the class of chitin synthase is not necessarily related to the function and importance of these genes among different fungal species.

To reveal the function of individual chitin synthase genes, double and triple disruption mutants were constructed and analysed. We previously found that the class II and class IV chitin synthases of $A$. nidulans have some overlapping function(s) in conidiation (Motoyama et al., 1996), and that the class I and class II chitin synthases of A. nidulans share important roles in hyphal growth and conidiation (Fujiwara et al., 2000). A partial redundancy of function of class II and class IV chitin synthases was also reported by another group (Culp et al., 2000). From these results, we speculated that chitin synthases in class I, class II and class IV may play important roles in growth or differentiation, and that these synthases cooperate with each other. In the yeast Saccharomyces cerevisiae, the class IV chitin synthase is involved in most of the cellular chitin synthesis and serves redundant functions with class II chitin synthase in growth (Valdivieso et al., 1991; Shaw et al., 1991). These results suggest that fungal chitin is synthesized by the overlapping and cooperative function of multiple chitin synthase genes.

In our previous study, we showed that $A$. nidulans $\operatorname{chs} D$, which encodes a class IV chitin synthase, is expressed in the growing hyphae (Motoyama et al., 1996), and chsB, which encodes a class III chitin synthase, plays essential roles in normal hyphal growth. These results suggest that ChsD and ChsB have some related functions in chitin synthesis and other activities of growing hyphae. In this study, we focused on the functional relationship between ChsB and ChsD in hyphal growth and in conidiation. The construction of double deletion mutants of $c h s B$ and $c h s D$ seemed to be difficult because null mutants of $c h s B$ are heavily damaged in hyphal growth (Yanai et al., 1994; Borgia et al., 1996). Therefore, we constructed conditional double mutants in which $c h s D$ was replaced with the $\arg B$ gene of $A$. nidulans, and $c h s B$ was placed under the control of a repressible promoter (the alcA promoter). We then analysed the functional relationship between the two chitin synthases.

\section{METHODS}

Strains, media and transformation. The A. nidulans strains used are shown in Table 1. Complete medium (CM) and minimal medium (MM) for A. nidulans described by Rowlands \& Turner (1973) were used. YGU medium $[0.5 \%$ yeast extract (Difco), $1 \%$ glucose, $0 \cdot 1 \%$ trace elements (Rowlands \& Turner 1973), $10 \mathrm{mM}$ uridine] was also used. When the expression of the alcA promoter was induced, we used $100 \mathrm{mM}$ threonine and $0 \cdot 1 \%$ fructose as carbon sources instead of glucose (MMFT). Transformation was done as described by May (1992). When the expression of the alcA promoter was induced in the transformation experiments, we used 1.2 M sorbitol as an osmotic stabilizer instead of sucrose. Transformants were grown in MM with appropriate supplements.

Plasmids were amplified in Escherichia coli MV1190 $[\Delta($ lacproAB) thi supE $\Delta(\operatorname{srl}-r e c A) 306:: \operatorname{Tn} 10\left(\right.$ Tet $\left.^{r}\right) \quad\left(\mathrm{F}^{\prime}\right.$ traD36 proAB lacl $\left.\left.{ }^{\mathrm{q}} \mathrm{Z} \Delta \mathrm{M} 15\right)\right]$. E. coli was grown in LB and transformation was done by standard methods (Sambrook et al., 1989).

Total DNA isolation and Southern analysis. Total DNA of filamentous fungi was extracted as described by Oakley et al. (1987). Southern analysis was done with ECL direct nucleic acid labelling and detection systems (Amersham Pharmacia Biotech). DNA was separated by electrophoresis on agarose gels and further manipulation was done as described in the manufacturer's instructions.

Total RNA isolation and Northern analysis. Total RNA was prepared from mycelia by using the RNeasy Mini Kit (QIAGEN). Five micrograms of total RNA were separated by electrophoresis on a formaldehyde-agarose gel, and blotted onto Hybond-XL membrane (Amersham Pharmacia Biotech) according to the manufacturer's instructions. Blots were hybridized to ${ }^{32} \mathrm{P}$-labelled probes prepared by using Random Primer DNA Labelling Kit Version 2 (TaKaRa). As a probe for $c h s B$ expression, the $1.6 \mathrm{~kb} \mathrm{NcoI}$ fragment from pchsB was used.

PCR-amplification of DNA fragments. PCR-amplification was done by using a thermal cycler (model 480, Perkin-Elmer/ Cetus) and Taq DNA polymerase (Boehringer Mannheim) or the Expand High Fidelity PCR system (Boehringer Mannheim).

RT-PCR amplification. Twenty micrograms of total RNA were treated with deoxyribonuclease (RT Grade) (NIPPON GENE), then reverse transcriptions were performed by using $3 \mu \mathrm{g}$ DNA-free RNA and ReverTra Ace reverse transcriptase (TOYOBO) according to the manufacturer's instructions. The primer used for reverse transcriptions was chsB.3072as (5'-GGCATCCAGTCTAGGTTGC-3'). For PCR amplifications, $5 \mu \mathrm{l}$ samples from $20 \mu \mathrm{l}$ of reverse transcription reactions were used. The primers used were chsB.2055s $\left(5^{\prime}\right.$-CATCATGGATCTGGTAGG-3') and chsB.3075as $\left(5^{\prime}-\right.$ ATACATACAATTGCAAGGC- $\left.3^{\prime}\right)$. Since we designed the primer set to amplify the region that contains an intron, the products of RT-PCR were expected to be $57 \mathrm{bp}$ smaller than the PCR products amplified from genomic DNA by using the same primer set. PCR products and $100 \mathrm{bp}$ DNA ladder (New England Biolabs) were electrophoresed on a $1.0 \%$ agarose gel.

Plasmid constructions. Plasmids used in this paper are shown in Table 2. To make the strain in which the expression of $c h s B$ is under the control of the alcA promoter, the plasmid p5BP- 
Table 1. A. nidulans strains used in this study

\begin{tabular}{|c|c|c|}
\hline Strain & Genotype & Source \\
\hline AU1 & pyrG89 wA3 $\operatorname{argB2}$ & Motoyama et al. (1994a) \\
\hline $\mathrm{AU} / \mathrm{A}$ & pyrG89 wA3 argB2 [pSS1] & This study \\
\hline ABPU1 & biA1 pyrG89 wA3 argB2 pyroA4 & Motoyama et al. (1996) \\
\hline $\mathrm{ABPU} / \mathrm{A}$ & biA1 pyrG89 wA3 argB2 pyroA4 [pSS1] & This study \\
\hline $\mathrm{ABPU} / \mathrm{AU}$ & biA1 pyrG89 wA3 argB2 pyroA4 [pSS1][pP1] & Motoyama et al. (1996) \\
\hline BM-3 & biA1 pyrG89 wA3 argB2 pyroA4 $\Delta$ chsB::pyr-4::alcA(p)::chsB & This study \\
\hline $\mathrm{BM}-3 / \mathrm{A} 1$ & biA1 pyrG89 wA3 argB2 pyroA4 $\Delta$ chsB::pyr-4::alcA(p)::chsB [pSS1] & This study \\
\hline BM-4 & biA1 pyrG89 wA3 argB2 pyro $A 4 \Delta c h s B::$ pyr-4::alcA(p)::chsB & This study \\
\hline BM-12 & biA1 pyrG89 wA3 argB2 pyroA4 $\Delta$ chsB::pyr-4::alcA(p)::chsB & This study \\
\hline BB-3 & biA1 pyrG89 wA3 argB2 pyroA4 $\Delta$ chsB::pyr-4::alcA(p)::chsB [pSS-chsB] & This study \\
\hline BD-2 & biA1 pyrG89 wA3 argB2 pyroA4 $\Delta$ chsB::pyr-4::alcA(p)::chsB $\Delta$ chsD::argB & This study \\
\hline D3-2 & biA1 pyrG89 wA3 $\operatorname{argB2}$ pyroA4 $\triangle$ chsD:: $\arg B$ & Motoyama et al. (1996) \\
\hline DB-13 & biA1 pyrG89 wA3 argB2 pyroA4 $\Delta$ chsB::pyr-4::alcA(p)::chsB $\Delta$ chsD::argB & This study \\
\hline DB-19 & biA1 pyrG89 wA3 argB2 pyroA4 $\Delta$ chsB::pyr-4::alcA(p)::chsB $\Delta$ chsD::argB & This study \\
\hline BL-1 & pyrG89 wA3 $\operatorname{argB2}[\mathrm{pB}-\mathrm{LAC}]$ & This study \\
\hline DL-4 & biA1 pyrG89 wA3 argB2 pyroA4 [pD-LAC] & This study \\
\hline
\end{tabular}

Table 2. Plasmids used in this study

\begin{tabular}{|c|c|c|}
\hline Plasmid & Construction & Reference \\
\hline pchsB & $\begin{array}{l}5 \cdot 5 \mathrm{~kb} \text { HindIII fragment containing the } c h s B \text { gene cloned into the HindIII site of } \\
\text { pUC118 }\end{array}$ & This study \\
\hline pchsBR & Identical to pchsB except that the direction of the $c h s B$ transcription is opposite & This study \\
\hline pchsB5.5H & $\begin{array}{l}\text { Plasmid derived from pchsBR by introducing a HindIII site just in front of the } c h s B \\
\text { initiation codon }\end{array}$ & This study \\
\hline pDJB1 & Plasmid containing the $p y r-4$ gene of $N$. crassa & Ballance \& Turner (1985) \\
\hline pDJB-ALC-B $\Delta$ & $\begin{array}{l}1.7 \mathrm{~kb} \text { HindIII-EcoRI fragment of the chsB N-terminal coding sequence downstream } \\
\text { of the alcA promoter cloned into pDJB1 }\end{array}$ & This study \\
\hline $\mathrm{pP} 1$ & Plasmid containing the $p y r-4$ gene of $N$. crassa & Motoyama et al. (1994a) \\
\hline p5BP & $\begin{array}{l}0.9 \mathrm{~kb} \text { EcoRI-HindIII fragment containing the } c h s B \text { promoter and } 5^{\prime} \text { noncoding } \\
\text { region cloned into the } \mathrm{X} b a \mathrm{I} \text { site of } \mathrm{pP} 1\end{array}$ & This study \\
\hline p5BP-ALCB & $\begin{array}{l}1.7 \mathrm{~kb} \text { ClaI fragment from } \mathrm{pDJB}-\mathrm{ALC}-\mathrm{B} \Delta \text { containing } \operatorname{alcA}(\mathrm{p}):: c h s B \text { cloned into the } \\
\text { Sse } 8387 \mathrm{I} \text { site of } \mathrm{p} 5 \mathrm{BP}\end{array}$ & This study \\
\hline pSS1 & $\begin{array}{l}1.8 \mathrm{~kb} \text { SalI-SphI fragment containing the } \operatorname{argB} \text { gene cloned into the SalI-SphI site of } \\
\text { pUC118 }\end{array}$ & Motoyama et al. (1994a) \\
\hline pSS-chsB & $5 \cdot 5 \mathrm{~kb}$ HindIII fragment containing the $c h s B$ gene cloned into the Bam HI site of pSS1 & This study \\
\hline pMC1871 & Plasmid containing the $l a c Z$ gene of E. coli & Casadaban et al. (1983) \\
\hline pSS-LZ & $\begin{array}{l}3.0 \mathrm{~kb} \text { Bam HI lacZ-containing fragment from pMC1871 cloned into the BamHI site } \\
\text { of pSS1 }\end{array}$ & This study \\
\hline pSS-TB-LZ & $\begin{array}{l}\text { Plasmid derived from pSS-LZ by introducing the terminator region of } c h s B \text { into the } \\
X b a \text { I site downstream of the lacZ gene }\end{array}$ & This study \\
\hline pB-LAC & $\begin{array}{l}\text { Construct to express the ChsB-LacZ fusion protein under the control of the } c h s B \\
\text { promoter; } 1 \cdot 8 \mathrm{~kb} \text { HindIII-NaeI fragment containing the } c h s B \text { promoter and leader } \\
\text { sequence was cloned into the SmaI site of pSS-TB-LZ }\end{array}$ & This study \\
\hline pchsD & $\begin{array}{l}7 \cdot 3 \mathrm{~kb} \text { Bam HI-SalI fragment containing the } c h s D \text { gene cloned into the BamHI-SalI } \\
\text { site of pUC119 }\end{array}$ & Motoyama et al. (1996) \\
\hline pD-LAC & $\begin{array}{l}\text { Construct to express the ChsD-LacZ fusion protein under the control of the } c h s D \\
\text { promoter; } 1.8 \mathrm{~kb} N s p \mathrm{~V} \text { fragment containing the } c h s D \text { promoter and leader } \\
\text { sequence was cloned into the } S m a \mathrm{I} \text { site of pSS-TB-LZ }\end{array}$ & This study \\
\hline $\mathrm{pD} \Delta \mathrm{A} 7$ & $\begin{array}{l}\text { Plasmid derived from pchsD by replacing the } 3.6 \mathrm{~kb} S p l \mathrm{I}-\mathrm{Xba \textrm {I }} \text { fragment containing } \\
\text { the entire coding region of } \operatorname{chs} D \text { with the } 1.8 \mathrm{~kb} \arg B \text {-containing fragment from } \\
\text { pSS1 }\end{array}$ & Motoyama et al. (1996) \\
\hline
\end{tabular}


ALCB was constructed as follows. The alcA promoter was amplified from the genomic DNA of strain AU1 by the PCR method using the two primers, M16-1 (5'-AAAATCGATGGCGGGGCGGAAATTGACA-3') and M16-2 (5'-AAAAAGCTTTGAGGCGAGGTGATAGGAT-3'), according to the sequence reported by Kulmburg et al. (1992). Using these two primers, 35 cycles were run consisting of a $94^{\circ} \mathrm{C} 1$ min melting step, a $56^{\circ} \mathrm{C} 2 \mathrm{~min}$ annealing step, and a $72{ }^{\circ} \mathrm{C} 3$ min extension step. The $5.5 \mathrm{~kb}$ HindIII fragment containing the $c h s B$ gene was prepared from the DNA purified from the phage clone that contains the $c h s B$ gene (Yanai et al., 1994) and ligated with HindIII-digested and bacterial alkaline phosphatase (AP)-treated pUC118 (Takara Shuzo) to yield pchsB and pchsBR. The direction of $c h s B$ transcription was from the HindIII site to the EcoRI site on the multiple cloning site of pchsB, and the direction of the $c h s B$ insert of pchsBR was reverse compared to pchsB.

The HindIII site (indicated by underlining, see below) was introduced on the plasmid pchsBR just in front of the initiation codon (indicated by boldface, see below) by a site-directed mutagenesis kit (Bio-Rad) using the primer KY-X (5'-GCCATGGTTAAGCTTGTATGTGC-3') to yield pchsB5.5H. The ClaI- and EcoRI-digested and AP-treated pDJB1 (Ballance \& Turner, 1985), the ClaI- and HindIII-digested $0.5 \mathrm{~kb}$ PCR fragment which contains the alcA promoter, and the $1.7 \mathrm{~kb}$ HindIII-EcoRI fragment which contains a region of $c h s B$, from its initiation codon to about half of ORF, derived from pchsB5.5H, were ligated to yield pDJB-ALC-B $\Delta$. The blunted $0.9 \mathrm{~kb}$ HindIII-EcoRI fragment, which contains the upstream region of the $c h s B$ promoter, derived from pchsB5.5H, was ligated with $\mathrm{XbaI}$-digested, blunted, and AP-treated $\mathrm{pP} 1$, a plasmid bearing the Neurospora crassa pyr4 gene (Motoyama et al., 1994a), to yield p5BP. The direction of $c h s B$ transcription was from the EcoRI site to the HindIII site on the multiple cloning site. The blunted $1.7 \mathrm{~kb}$ ClaI fragment, which contains the alcA promoter and part of the $c h s B$ ORF, derived from pDJB-ALC-B $\Delta$, was ligated with Sse8387I-digested, blunted, and AP-treated p5BP to yield p5BP-ALCB.

The plasmid pSS-chsB, for $c h s B$ expression, was constructed by ligating the $5 \cdot 5 \mathrm{~kb}$ HindIII fragment, which contains the $c h s B$ gene derived from pchsB, and BamHI-digested, blunted, and AP-treated pSS1 (Motoyama et al., 1994a).

Plasmids for the analysis of promoter activities in A. nidulans with lacZ of E. coli as a reporter gene were constructed as follows. The $3.0 \mathrm{~kb}$ Bam HI fragment which contains lacZ of E. coli derived from pMC1871 (Casadaban et al., 1983) was ligated with BamHI-digested and AP-treated pSS1 to yield pSS-LZ. The blunted $0.9 \mathrm{~kb} S p h \mathrm{I}$ fragment, which is expected to contain the terminator region of $c h s B$, derived from pchsB5.5H, was ligated with $\mathrm{Xba \textrm {I }}$-digested, blunted, and APtreated pSS-LZ to yield pSS-TB-LZ. The $1.8 \mathrm{~kb}$ HindIII-NaeI fragment, which contains the $c h s B$ promoter $(1.3 \mathrm{~kb})$ and a part of the chsB ORF $(0.5 \mathrm{~kb})$, derived from pchsB, was blunted and ligated with SmaI-digested and AP-treated pSSTB-LZ to yield pB-LAC. The $1.8 \mathrm{~kb} \mathrm{NspV}$ fragment, which contains the $c h s D$ promoter $(1.4 \mathrm{~kb})$ and a part of the $c h s D$ $\operatorname{ORF}(0.4 \mathrm{~kb})$, derived from pchsD, was blunted and ligated with SmaI-digested and AP-treated pSS-TB-LZ to yield pDLAC. Linkage points were confirmed by sequencing with a primer, prm-LZ2 (5'-TTCTGGTGCCGGAAACCA-3'). We found some sequencing errors in the $c h s D$ and the new version of the $c h s D$ nucleotide sequence is registered with accession no. D83246. Consequently, total amino acid residues of ChsD were changed from 1086 to 1184 due to the extension of 96 aa residues in its $\mathrm{N}$-terminus. From the results of $5^{\prime}$-RACE
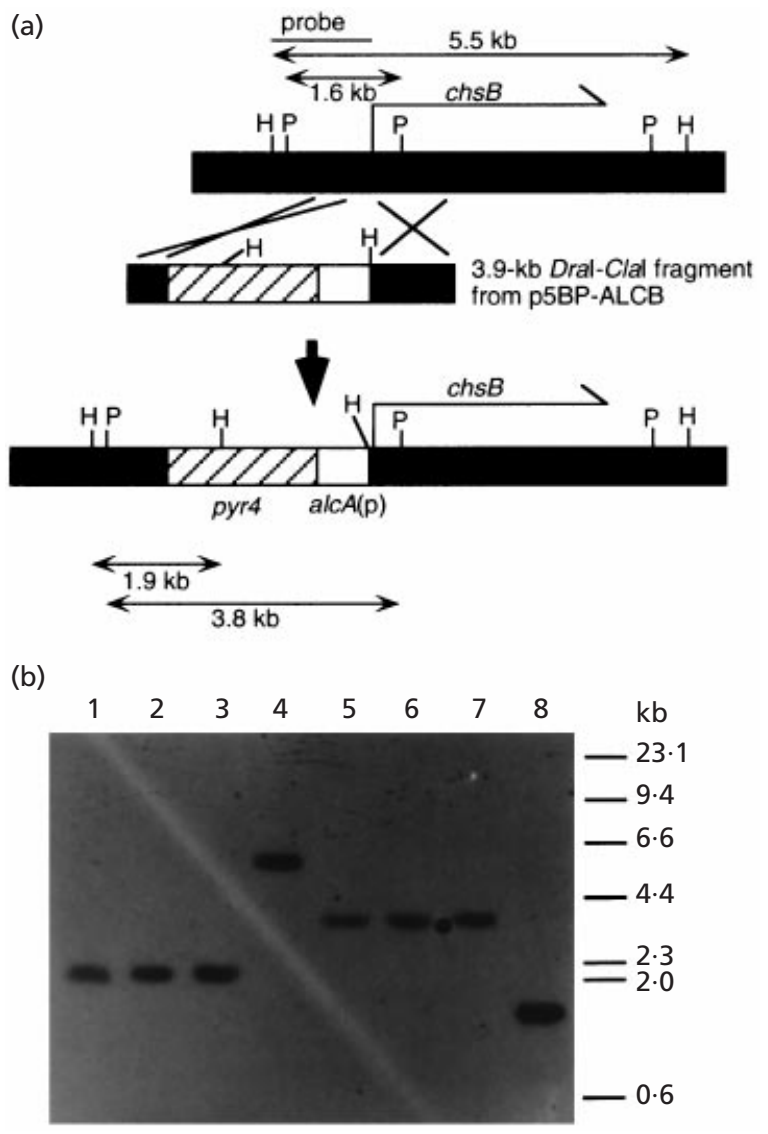

Fig. 1. Constructions of conditional chsB mutant strains. (a) Construction of strains BM-3, BM- 4 and $B M-12$ by inserting the $3.9 \mathrm{~kb}$ Dral-Clal fragment from p5BP-ALCB into the promoter region of chsB. Abbreviations: H, HindIII; P, Pstl. (b) Southern analysis of transformants. HindIII-digested total DNA of strain BM-3 (lane 1), strain BM-4 (lane 2), strain BM-12 (lane 3) and strain ABPU1 (lane 4) was hybridized with the $1.3 \mathrm{~kb}$ HindIII fragment (Fig. 1a, probe) from pchsB5.5H. Pstl-digested total DNA of strain BM-3 (lane 5), strain BM-4 (lane 6), strain BM-12 (lane 7) and strain ABPU1 (lane 8) was hybridized with the same $1.3 \mathrm{~kb}$ HindIII fragment.

experiments, the transcriptional start point(s) were estimated approximately $530 \mathrm{bp}$ upstream of the revised ATG initiation codon and an intron of $151 \mathrm{bp}$ was identified from -6 to -157 when the first A of the revised initiation codon was designated +1 .

Construction of $\boldsymbol{A}$. nidulans strains by transformation. All the strains originated from AU1 or ABPU1. The $\arg B$ gene of $A$. nidulans and the pyr4 gene of $N$. crassa were used as selectable markers to complement arginine- and uridine-auxotrophy, respectively.

Conditional mutants of $c h s B$ were made as follows. The promoter of $c h s B$ in the genome of $A$. nidulans strain ABPU1 was interrupted with the alcA promoter by transformation with the $3.9 \mathrm{~kb}$ DraI-ClaI fragment from p5BP-ALCB. By Southern analysis of HindIII-digested or PstI-digested total DNA of ABPU1 transformants probed with the $1.3 \mathrm{~kb}$ HindIII fragment (Fig. 1a, probe) from pchsB5.5H, homologous recombination at the chsB locus was confirmed in strains 


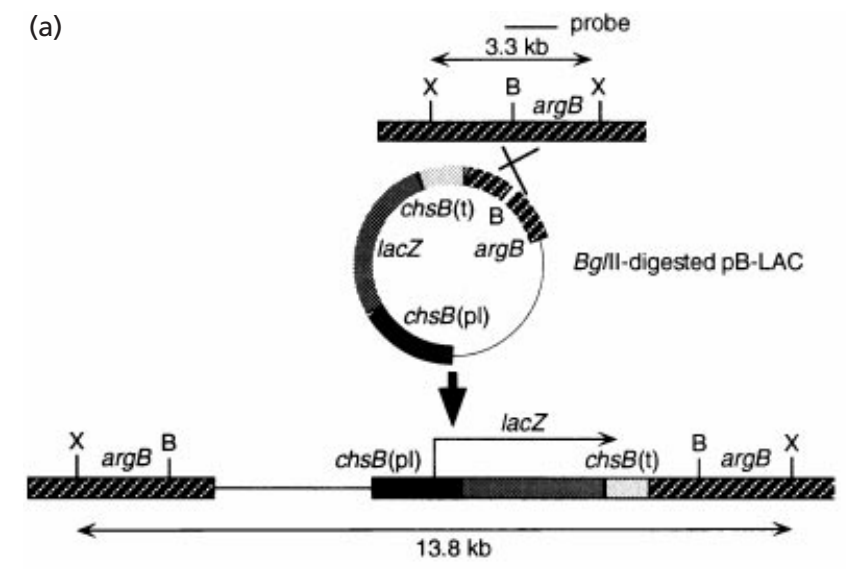

(b)

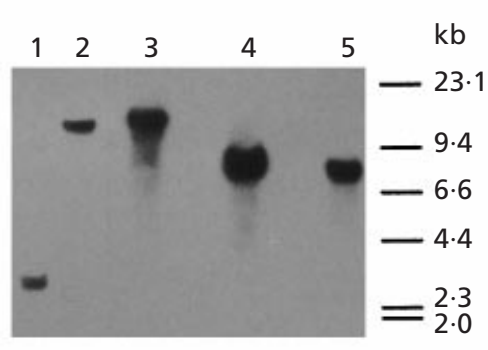

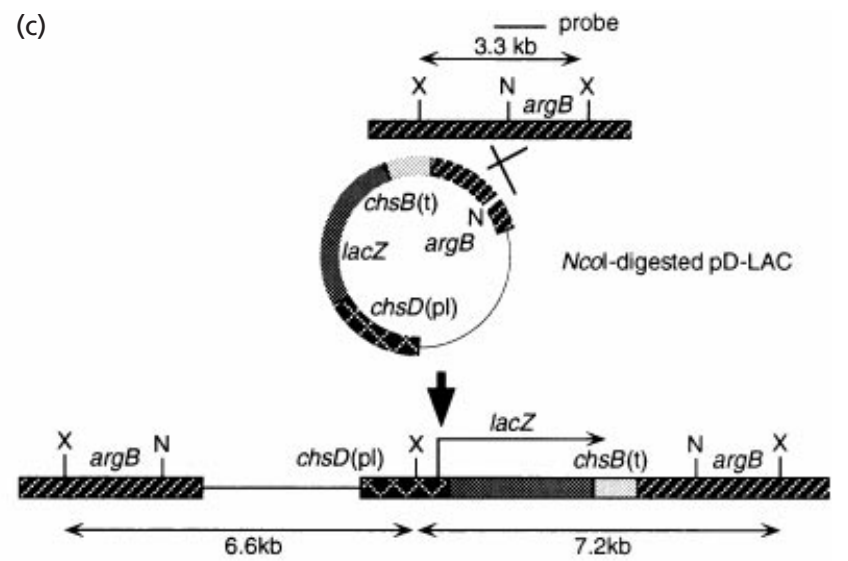

(d)

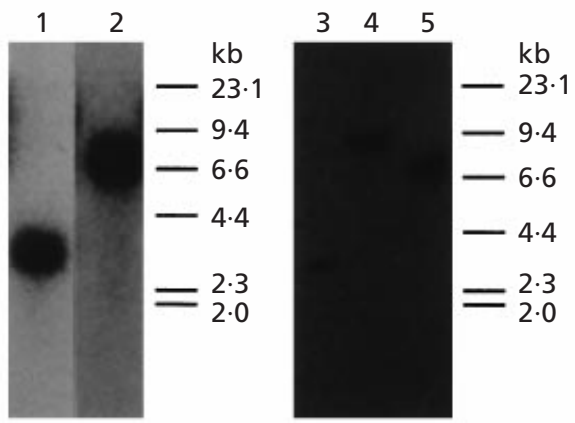

Fig. 2. Construction of strains to analyse promoter activities of $c h s B$ and $c h s D$. (a) Construction of strain $B L-1$, which produces $\beta$-galactosidase under the control of the chsB promoter. Strain AU1 was transformed with Bg/ll-digested pBLAC. Abbreviations: B, Bg/ll; X, Xbal. (b) Southern blot analysis of Xbal-digested (lanes 1-3), Stul-digested (lane 4) and HindIII-digested (lane 5) total DNAs from strain AU1 (lane 1) and from strain BL-1 (lanes 2-5) probed with the $1 \cdot 1 \mathrm{~kb}$ Stul-HindllI fragment from pSS1 (lanes 1 and 2) or probed with the $3.0 \mathrm{~kb} \mathrm{BamHI}$ fragment of lacZ from pMC1871 (lanes $3-5)$. (c) Construction of strain DL-4, which produces $\beta$-galactosidase under the control of the chs $D$ promoter. Strain ABPU1 was transformed with Ncol-digested pD-LAC. Abbreviations: N, Nco I; X, Xbal. (d) Lanes 1 and 2, Southern blot analysis of Xbal-digested total DNA from strains ABPU1 (lane 1) and DL-4 (lane 2) probed with the $1.1 \mathrm{~kb}$ Stul-HindIII fragment from pSS1. Lanes 3-5, Southern blot analysis of BamHI- (lane 3), HindIII- (lane 4) and Xbal- (lane 5) digested total DNA of strain DL-4 probed with the $3.0 \mathrm{~kb}$ fragment from pSS-TB-LZ.

named BM-3, BM-4 and BM-12 by a shift of the signal size from $5.5 \mathrm{~kb}$ to $1.9 \mathrm{~kb}$ and from $1.6 \mathrm{~kb}$ to $3.8 \mathrm{~kb}$, respectively (Fig. 1b, lanes 1-8). By the same strategy, the promoter of $c h s B$ in the genome of the chsD null mutant D3-2 (Table 1, Motoyama et al., 1996) was exchanged with the alcA promoter to yield strains DB-13 and DB-19. The exchange of these promoters was confirmed by Southern analysis (data not shown). Strain BB-3 was created by transformation of strain BM-3 with pSS-chsB. The strains ABPU/A and BM-3/A1 were constructed by transformation of strains ABPU1 and BM-3, respectively, with pSS1. chsD of strain BM-3 was replaced with the $\arg B$ gene by using $\mathrm{pD} \Delta \mathrm{A} 7$ as described previously (Motoyama et al., 1996), generating a strain designated as BD-2.

The strain to analyse the promoter activity of $c h s B$ was constructed as follows. AU1 was transformed with BglIIdigested pB-LAC. By Southern analysis of XbaI-digested total DNA of a transformant, BL-1, probed with the $1 \cdot 1 \mathrm{~kb}$ HindIII-StuI fragment (Fig. 2a, probe) from pSS1, homologous recombination at the $\arg B$ locus was confirmed by a shift of the signal size from $3.5 \mathrm{~kb}$ to $14 \mathrm{~kb}$ (Fig. 2b, lane 2). Only one signal was found by Southern analysis of total DNA of this transformant digested with three different restriction enzymes and probed with the $3.0 \mathrm{~kb} B a m \mathrm{HI}$ fragment which contains lacZ from pMC1871, indicating that one copy of the plasmid was integrated into this transformant (Fig. 2b, lanes 3-5). The strain to analyse the promoter activity of $c h s D$ was constructed as follows. ABPU1 was transformed with NcoIdigested $\mathrm{pD}$-LAC. By Southern analysis of XbaI-digested total DNA of a transformant, DL-4, probed with the $1 \cdot 1 \mathrm{~kb}$ HindIII-StuI fragment (Fig. 2c, probe) from pSS1, homologous recombination at the $\arg B$ locus was confirmed by a shift of the signal size from $3 \cdot 5 \mathrm{~kb}$ to $6 \cdot 6 \mathrm{~kb}$ and $7 \cdot 2 \mathrm{~kb}$ (Fig. $2 \mathrm{~d}$, lane 2). Only one signal was found by Southern analysis of total DNA of this transformant digested with three different restriction enzymes and probed with the $3.0 \mathrm{~kb} B a m \mathrm{HI}$ fragment which contains lacZ from pSS-TB-LZ, indicating that one copy of the plasmid was integrated into this transformant (Fig. $2 \mathrm{~d}$, lanes 3-5).

Calcofluor white staining. Conidia were spread on a YGU plate, and incubated for $24 \mathrm{~h}$ at $37^{\circ} \mathrm{C}$. Hyphae on a piece of agar (approx. $15 \mathrm{~mm} \times 15 \mathrm{~mm}$ ) were cut off, stained with $0.01 \%$ calcofluor white (fluorescent brightener 28, Sigma), and observed under a fluorescent microscope. 
Carbon source exchange experiment. Conidial suspensions of A. nidulans were inoculated into liquid MMFT $(200 \mathrm{ml}$, fructose and threonine as carbon sources) in a $500 \mathrm{ml}$ Erlenmeyer flask to a final concentration of $2 \times 10^{6}$ conidia $\mathrm{ml}^{-1}$. After agitating the flasks on a rotary shaker for $17 \mathrm{~h}$ at $37^{\circ} \mathrm{C}$, mycelia were collected by filtration through G3 glass filter, washed by liquid MM (glucose as a carbon source), and spread on MM plates (glucose as a carbon source). After $25 \mathrm{~h}$ incubation at $37^{\circ} \mathrm{C}$, conidia were collected from the plates and the numbers were counted using a haemocytometer. All the MMFT and MM used were supplemented with $0.20 \mathrm{mg}$ arginine $\mathrm{ml}^{-1}, 0.02 \mu \mathrm{g}$ biotin $\mathrm{ml}^{-1}, 0.50 \mu \mathrm{g}$ pyridoxine $\mathrm{ml}^{-1}$ and $10 \mathrm{mM}$ uridine. To examine the abundance of the $c h s B$ transcript, total RNA was prepared from BM-3/A1 (chsB mutant) mycelia taken at $0,5,15$ and $25 \mathrm{~h}$ after transfer onto a MM plate.

Chitin content determination. Chitin content was determined as described previously (Fujiwara et al., 2000), except that strains were grown in $100 \mathrm{ml} \mathrm{YGU}$ medium for $18 \mathrm{~h}$ at $37^{\circ} \mathrm{C}$, and that the cell-wall-containing pellets were sonicated in distilled water to a fine suspension before enzymic treatment. The protein levels, by which we normalized the amount of $\mathrm{N}$-acetylglucosamine, were not very different between the cell extracts from different strains.

In situ staining of the $\boldsymbol{\beta}$-galactosidase activity. $\beta$-Galactosidase activity was stained in situ by a modification of a method reported previously (Adams \& Timberlake, 1990; Aguirre et al., 1990). In brief, $20 \mu \mathrm{l}$ of a conidial suspension $\left(10^{8}\right.$ conidia $\left.\mathrm{ml}^{-1}\right)$ was spread on a MM plate (supplemented with biotin, pyridoxine and uridine). After incubating for 13 or $36 \mathrm{~h}$ at $37^{\circ} \mathrm{C}$, hyphae on a piece of agar (approx. $15 \mathrm{~mm} \times 15 \mathrm{~mm}$ ) were cut off, and treated with chloroform vapour for $20 \mathrm{~min}$ at room temperature. The piece was transferred into a staining solution $(0.05 \mathrm{M}$ sodium phosphate, $\mathrm{pH} 7.5,0.02 \% \mathrm{X}-\mathrm{Gal})$, incubated for $2 \mathrm{~h}$ at $37^{\circ} \mathrm{C}$, and cells were observed under a microscope.

Microscopy. Samples were observed and photographed by using a light microscope (model BHS-RFK, Olympus) equipped with an automatic camera (model PM-10ADS, Olympus) attachment.

\section{RESULTS}

\section{Construction of conditional mutants of chsB}

We constructed $c h s B$-conditional mutants in which $c h s B$ was placed under an inducible promoter, namely the alcohol dehydrogenase gene $(\operatorname{alc} A)$ promoter of $A$. nidu- lans (Kulmburg et al., 1992), and we analysed the functions of ChsB using these mutants. In these mutants, the expression of $c h s B$ is expected to be induced by ethanol or threonine and repressed by glucose. By introducing the $3.9 \mathrm{~kb} \mathrm{DraI-ClaI}$ fragment from p5BPALCB into strain ABPU1, we obtained three transformants in which the promoter of $c h s B$ in the genome was interrupted by the alcA promoter. We designated these three transformants BM-3, BM-4 and BM-12 (Fig. 1, Table 1). We used strain BM-3 for further experiments.

\section{Effect of chsB repression on growth and differentiation}

We analysed the effect of the repression of $c h s B$ expression on the growth of $A$. nidulans by comparing the radial growth rate of the $c h s B$-repressed strain with that of the wild-type strain. Since auxotrophic markers could affect the growth rate, BM-3/A1 was constructed by the transformation of BM-3 with pSS1, a plasmid containing the $\arg B$ gene, and the growth rate of $\mathrm{BM}$ $3 / \mathrm{A} 1$ was compared with that of the wild-type strain ABPU/AU. Conidia of each strain were point-inoculated on solid medium and incubated for $72 \mathrm{~h}$ at $37^{\circ} \mathrm{C}$. The colony diameter was then determined (Table 3). On $\mathrm{MM}$ plates $[\operatorname{alc} A(\mathrm{p})$-repressing condition], the colony diameter of BM-3/A1 (15.5 $\pm 4 \cdot 3 \mathrm{~mm})$ was much smaller than that of ABPU/AU $(34.5 \pm 2.9 \mathrm{~mm})$. Under this condition, dark brown pigmentation of hyphae in the agar was observed in BM-3/A1. Borgia et al. (1996) reported that the $c h s B$ disruptant colonies became brown. Significant reduction in the growth rate of BM-3/A1 was also observed on YGU plates $[\operatorname{alcA}(\mathrm{p})$ repressing condition]. Since the growth defects of some cell-wall-deficient mutants can occasionally be remedied by the addition of osmotic stabilizers into the media, we examined the effects of osmotic stabilizers. The growth defect of strain BM-3/A1 on YGU plates was not suppressed by the addition of $0.6 \mathrm{M} \mathrm{KCl}$. On MMFT plates $[\operatorname{alc} A(\mathrm{p})$-inducing condition], the colony diameter of BM-3/A1 $(31.8 \pm 0.8 \mathrm{~mm})$ was slightly smaller than that of $\mathrm{ABPU} / \mathrm{AU}(36.7 \pm 0.9 \mathrm{~mm})$. Taken together, these results suggest that the growth defect of BM-3/A1 on MM and YGU plate was caused by the repression of chsB expression.

Table 3. Comparison of growth rate of the wild-type strains and mutants

\begin{tabular}{|c|c|c|c|c|c|}
\hline \multirow[t]{2}{*}{ Strain } & \multirow[t]{2}{*}{ Relevant genotype } & \multicolumn{4}{|c|}{ Colony diameter $(\mathrm{mm})(\text { means } \pm \mathrm{SD})^{*}$} \\
\hline & & MMFT & MM & YGU & $\mathrm{YGU}+0.6 \mathrm{M} \mathrm{KCl}$ \\
\hline $\mathrm{ABPU} / \mathrm{AU}$ & Wild type & $36 \cdot 7 \pm 0 \cdot 9$ & $34 \cdot 5 \pm 2 \cdot 9$ & $35 \cdot 7 \pm 2 \cdot 6$ & $47 \cdot 3 \pm 0 \cdot 9$ \\
\hline BM-3/A1 & $\operatorname{alc} A(\mathrm{p}):: \operatorname{chs} B$ & $31 \cdot 8 \pm 0 \cdot 8$ & $15 \cdot 5 \pm 4 \cdot 3$ & $12 \cdot 1 \pm 2 \cdot 7$ & $12 \cdot 3 \pm 2 \cdot 5$ \\
\hline DB-13 & $\operatorname{alc} A(\mathrm{p}):: \operatorname{chs} B \Delta c h s D$ & $37 \cdot 2 \pm 1 \cdot 4$ & $7 \cdot 3 \pm 0 \cdot 2$ & $10 \cdot 2 \pm 0 \cdot 2$ & $5 \cdot 9 \pm 0 \cdot 3$ \\
\hline $\mathrm{ABPU} / \mathrm{A}$ & pyrG & $31 \cdot 7 \pm 1 \cdot 2$ & $29 \cdot 0 \pm 0 \cdot 8$ & $29 \cdot 7 \pm 1 \cdot 2$ & $40 \cdot 3 \pm 0 \cdot 9$ \\
\hline D3-2 & $\Delta c h s D$ pyrG & $34 \cdot 7 \pm 0 \cdot 9$ & $35 \cdot 2 \pm 0 \cdot 6$ & $33 \cdot 8 \pm 0 \cdot 2$ & $44 \cdot 5 \pm 0 \cdot 4$ \\
\hline
\end{tabular}

*Strains were grown for $72 \mathrm{~h}$ at $37^{\circ} \mathrm{C}$. Means \pm SD were calculated from three independent experiments. 
We next analysed the morphology of BM-3/A1 under the chsB-repressing condition. BM-3/A1, ABPU/AU and BB-3, an alcA $(\mathrm{p}):: c h s B$ strain bearing an extra copy of the intact $c h s B$ gene, were incubated for $24 \mathrm{~h}$ on YGU plates. Hyphal growth and conidiophore development were then observed (Fig. 3). BM-3/A1 displayed defects in hyphal growth (Fig. 3d, e); specifically, its hyphae branched more frequently than those of ABPU/AU, and the positions of branch formation were aberrantly close to the tips of the hyphae or to each other. The lateral walls lacked smoothness. Hyphal tips, branching points close to tips, and septa, which appeared to be formed normally, were intensely stained with calcofluor white, which is known to bind to chitin (Fig. 3e). Conidiophores developed rather normally on BM-3/A1 colonies, although fewer of them and of the conidia produced on each of them were produced than in ABPU/AU (Fig. 3f, and data not shown). The morphology of BB-3 was almost the same as that of ABPU/AU (Fig. 3i, j, k). These results suggest that the defects of BM-3/A1 were caused by the repression of the $c h s B$ expression.

\section{Involvement of chsB in conidiation}

In the previous section, we showed that BM-3/A1 has some defects in asexual development. It is possible that ChsB is directly involved in conidiophore development and conidia formation, or that ChsB is involved only in hyphal growth. In the latter case, the defect in conidiophores and conidia formation could be caused by the partial inability to produce conidiation-competent hyphae (Miller et al., 1991). To clarify which possibility is the case, we performed a carbon source exchange experiment (see Methods). Conidiation-competent hyphae of ABPU/AU, BM-3/A1 and BB-3, cultured in alcA(p)-inducing liquid MMFT, were transferred onto alcA $(\mathrm{p})$-repressing $\mathrm{MM}$ plates on which conidiation was induced. The numbers of conidia, which were expected to be produced without additional chsB expression, were then counted (Table 4). BM-3/A1 could form only $45 \%$ of the number of conidia produced by ABPU/AU. The efficiency of the conidiation of BB-3 was recovered up to $75 \%$ of that of ABPU/AU, suggesting that a part of the reduction in the conidiation efficiency of BM-3/A1 could be due to the absence of $c h s B$ expression during developmental induction. The reason why the conidiation efficiency of BB-3 could not reach the same level as that of the wild-type strain is not clear. It is possible that the expression of the extra copy of $c h s B$ is not sufficient due to the effect of the position where $c h s B$ was integrated.

In comparison with the previous report by Borgia et al. (1996), in which they showed that the chsB disruptants did not conidiate, the reduction in conidiation efficiency of BM-3/A1 was rather moderate. It is possible that the repression of $c h s B$ transcription is incomplete on MM plates, and the incompleteness could relieve the conidiation defect to some extent. We examined the abundance of the $c h s B$ transcripts after the shift to MM plate by Northern analysis (Fig. 4a). chsB mRNA was easily
Table 4. Conidia formation efficiency of the wild-type strain and mutants

\begin{tabular}{|llc|}
\hline Strain & Relevant genotype & $\begin{array}{c}\mathbf{1 0}^{-4} \times \text { No. of conidia } \\
\left.\mathbf{m m}^{-2} \text { (mean } \pm \mathrm{SD}\right)^{*}\end{array}$ \\
\hline ABPU/AU & Wild-type & 100 \\
BM-3/A1 & alc $A(\mathrm{p}):: \operatorname{chs} B$ & $45 \pm 4 \cdot 9$ \\
BB-3 & alc $A(\mathrm{p}):: \operatorname{chs} B$ chs $B$ & $75 \pm 3 \cdot 9$ \\
DB-13 & alc $A(\mathrm{p}):: \operatorname{chs} B \Delta \operatorname{chs} D$ & $26 \pm 1 \cdot 8$ \\
\hline
\end{tabular}

* Means $\pm S D$ were calculated from at least three independent experiments.

detectable in the sample prepared from the mycelium cultured for $17 \mathrm{~h}$ in liquid MMFT (Fig. 4a, 0 h). After shift to a MM plate, $c h s B$ mRNA decreased drastically by $5 \mathrm{~h}$ after shift, and was almost undetectable at $15 \mathrm{~h}$ after shift (Fig. 4a). At 25 h after shift, a low level of $c h s B$ expression was again detected (Fig. 4a). This resumption of $c h s B$ expression may be due to the decrease of glucose level and the concomitant derepression of the alcA promoter. By performing RT-PCR, we obtained a clearer result that shows the presence of the $c h s B$ transcripts at $5 \mathrm{~h}$ and $25 \mathrm{~h}$ after shift (Fig. $4 \mathrm{~b}$ ). These results imply that the conidiation defect of BM-3/A1 was underestimated in the carbon source exchange experiment because of the stability of $c h s B$ mRNA and the leaky expression of $c h s B$.

\section{Functional importance of ChsD in the chsB- conditional mutant}

To examine the functional importance of ChsD under the ChsB-repressing condition, we generated double mutants of $c h s B$ and $c h s D$. We transformed the $c h s D$ null mutant, D3-2 (Motoyama et al., 1996), by using the same strategy as the BM-3 construction, generating strains DB-13 and DB-19 (see Methods). Since DB-19 showed almost the same phenotype as DB-13 (data not shown), data on DB-13 are given for the following experiments. We also generated a double mutant by deleting the chsD gene of BM-3. The resulting strain, $\mathrm{BD}-2$, exhibited almost the same phenotype as DB-13 under the conditions tested (data not shown). We first examined the growth rate of the double mutant (Table 3). On MMFT plates, the colony diameter of DB-13 was almost identical to that of the wild-type strain ABPU/AU and was larger than that of BM-3/A1. The colony diameter of the double mutant was about half that of BM-3/A1 on MM plates, whereas it was almost the same as that of BM-3/A1 on plates of YG, another alc $A(\mathrm{p})$-repressing medium. We found the osmolarity of YGU medium to be lower than that of minimal medium (data not shown). Supplementing YGU plates with $0.6 \mathrm{M} \mathrm{KCl}$ caused the colony diameter of DB-13 to decrease to half of that of BM-3/A1. The colony diameters of the $c h s D$ null mutant (D3-2) were slightly larger than those of the relevant wild-type strain (ABPU/A) under all conditions tested. These results indicate that the importance of the ChsD function in 

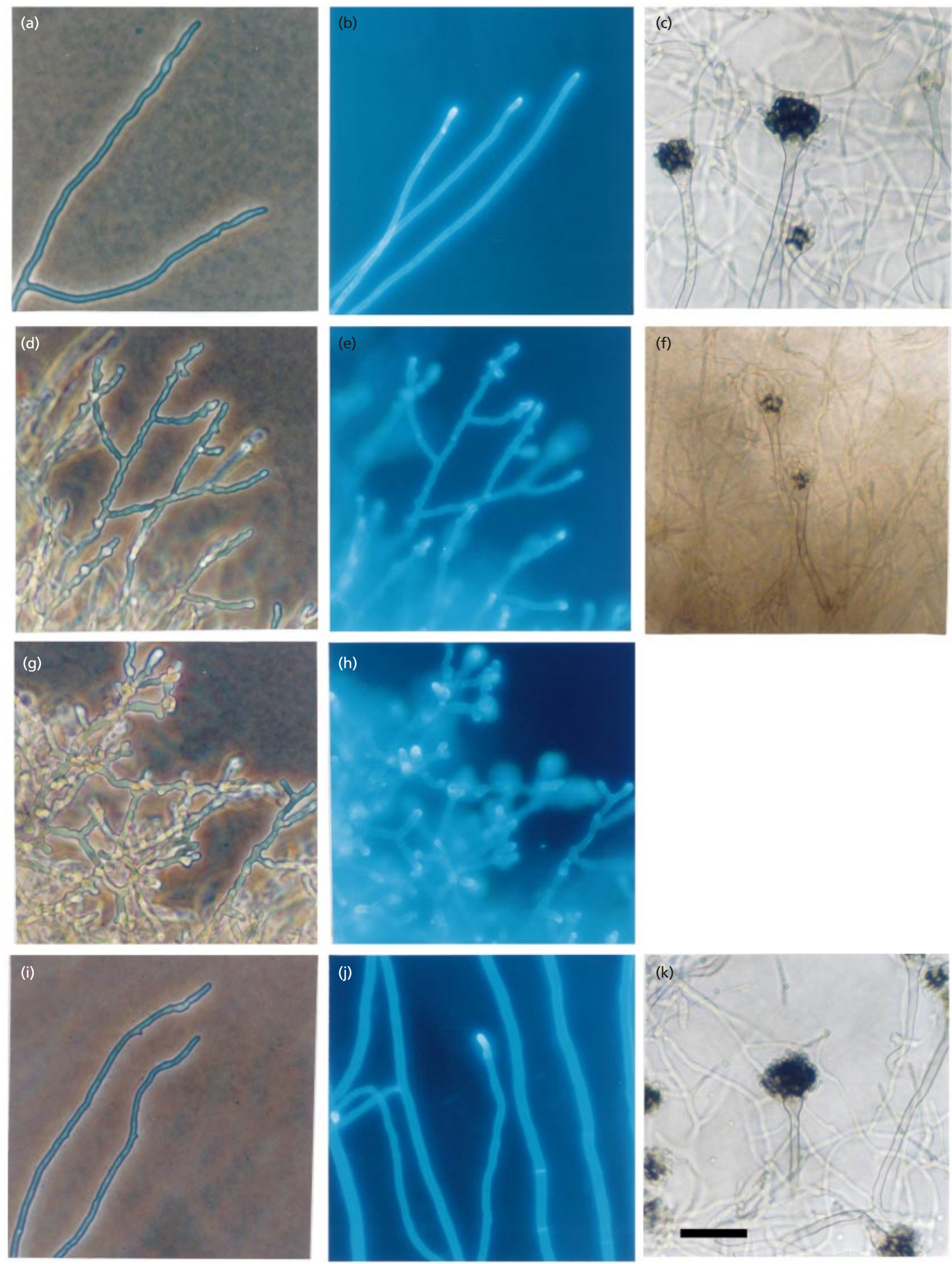

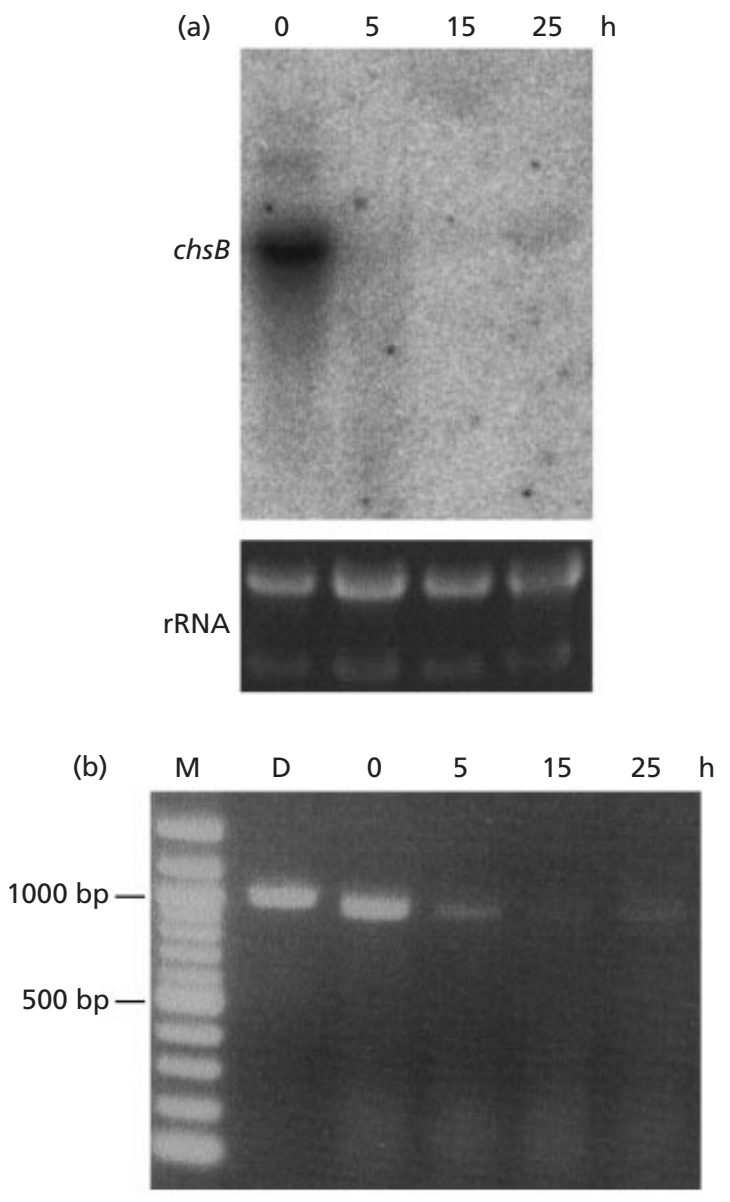

Fig. 4. Expression of $c h s B$ after the shift from inducing conditions to repressing conditions. (a) Northern blot analysis of chsB. Total RNA was prepared from the mycelia of the chsB mutant (BM-3/A1) cultured for 0, 5, 15 and $25 \mathrm{~h}$ after the shift onto MM plates. Equal loading of total RNA was evaluated by ethidium bromide staining ( $r R N A$ ). (b) RT-PCR amplification of $c h s B$. The RNA samples used for Northern analysis were treated with DNase, and subjected to RT-PCR. To assure the amplification from CDNA, PCR was also performed by using total DNA from strain ABPU1 (wild-type) as a template (lane D). The DNA standard (100 bp ladder) is in lane M.

hyphal growth increased under high osmolarity conditions when the expression of $c h s B$ was repressed.

When the hyphal morphology of DB-13 on YGU plate was observed microscopically, the hyphae were seen to be even more disorganized than those of BM-3/A1 (Fig. $3 \mathrm{~g}, \mathrm{~h}$ ). The number of conidiophores was much smaller than that of BM-3/A1 (data not shown). The number was not greatly increased by $24 \mathrm{~h}$ of additional incubation (data not shown).

In the carbon source exchange experiment, it was shown that DB-13 generated approximately $60 \%$ of the number
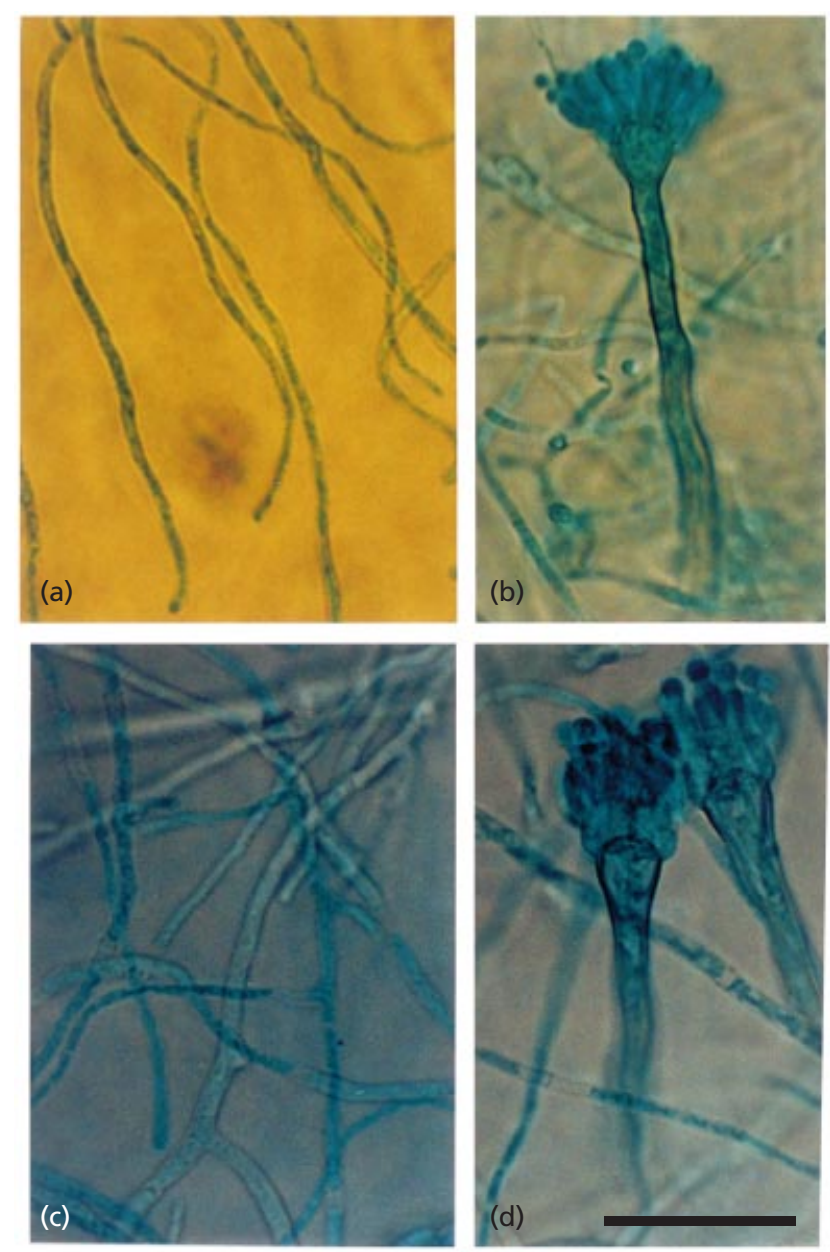

Fig. 5. Spatial expression patterns of $c h s B$ and $c h s D$. Conidia from strains BL-1 $(a, b)$ and DL-4 (c, d) were incubated for $13 \mathrm{~h}$ $(a, c)$ or for $36 \mathrm{~h}(b, d)$ on MM plates, and mycelia were stained with X-Gal and observed under a microscope. Bar, $25 \mu \mathrm{m}$.

of conidia generated by BM-3/A1 (Table 4). Taking into account that the $c h s D$ single mutants show no significant reduction in conidiation efficiency (Motoyama et al., 1996; Culp et al., 2000, in which chsD is referred to as chsE), it is suggested that the importance of the ChsD function in conidiation also increased under the $c h s B$ repressing condition.

We measured the chitin content of BM-3/A1 and DB-13 (Table 5). The chitin contents of BM-3/A1 in YGU and in YGU containing $0.6 \mathrm{M} \mathrm{KCl}$ were both approximately $70 \%$ of those of the wild-type strain (ABPU/AU), whereas those of BB-3 were almost the same as those of the wild-type strain. The chitin contents of DB- 13 were approximately half those of BM-3/A1. Since the chitin contents of D3-2 under these conditions were not very different from those of the relevant wild-type strain

Fig. 3. Morphology of the chsB single mutant and the $c h s B$ chsD double mutant. The wild-type strain (ABPU/AU) (a-c), the chsB mutant (BM-3/A1) (d-f), the chsB chsD double mutant (DB-13) $(g, h)$ and the chsB mutant transformed with the chsB gene (BB-3) (i-k) were grown for $24 \mathrm{~h}$ on YGU plates. Hyphae were stained with calcofluor white to visualize chitin distribution (b, e, h and j). Bar, $30 \mu \mathrm{m}$. 
Table 5. Chitin contents of the wild-type strains and mutants

\begin{tabular}{|c|c|c|c|}
\hline \multirow{2}{*}{ Strain } & \multirow[t]{2}{*}{ Relevant genotype } & \multicolumn{2}{|c|}{ Chitin content $\left[\mathrm{mg} \text { GlcNAc }(\mathrm{mg} \text { protein })^{-1}\right]^{*}$} \\
\hline & & YGU & $\mathrm{YGU}+0.6 \mathrm{M} \mathrm{KCl}$ \\
\hline $\mathrm{ABPU} / \mathrm{AU}$ & Wild-type & $1.09(100) \pm 0.090$ & $0.79(100) \pm 0.031$ \\
\hline BM-3/A1 & $\operatorname{alc} A(\mathrm{p}):: \operatorname{chs} B$ & $0.73(67) \pm 0.038$ & $0.59(75) \pm 0.031$ \\
\hline DB-13 & $\operatorname{alcA}(\mathrm{p}):: c h s B \Delta c h s D$ & $0.37(34) \pm 0.004$ & $0 \cdot 31(39) \pm 0 \cdot 022$ \\
\hline BB-3 & $\operatorname{alc} A(\mathrm{p}):: \operatorname{chs} B \operatorname{chs} B$ & $1.04(95) \pm 0.040$ & $0.77(97) \pm 0.064$ \\
\hline $\mathrm{ABPU} / \mathrm{A}$ & pyrG & $0.80(100) \pm 0.053$ & $0 \cdot 74(100) \pm 0 \cdot 043$ \\
\hline D3-2 & $\Delta c h s D$ pyrG & $0.74(93) \pm 0.031$ & $0.63(85) \pm 0.008$ \\
\hline
\end{tabular}

* Means \pm SD were calculated from the results of triplicate samples. Similar results were reproducibly observed in several experiments. The ratio of chitin content of each strain to that of each of the wildtype strain is shown in parentheses.

(ABPU/A), the effect of the chsD deletion on chitin content was considered to be larger in the chsBconditional mutant than in the wild-type strain. Taking these results together, we concluded that the chsB repression increased the importance of the ChsD functions in hyphal growth, conidiation and chitin content maintenance.

\section{Expression of chsB and chsD}

Transcriptional regulation of chitin synthase genes has been shown in other fungi (Chen-Wu et al., 1992; Pammer et al., 1992; Sudoh et al., 1993; Choi et al., 1994; Motoyama et al., 1994b; Xoconostle-Cázares et al., 1996, 1997; Munro et al., 1998; Wang \& Szaniszlo, 2000), and seems to be one of the regulatory mechanisms for chitin synthesis. To investigate the involvement of ChsB and ChsD in hyphal growth and conidiation in another way, we analysed the expression pattern of $c h s B$ and $c h s D$ using lacZ of $E$. coli as a reporter gene. We constructed strains BL-1 and DL-4 using plasmids pBLAC and pD-LAC, respectively (Fig. 2, see Methods). In strain BL-1, it was predicted that the ChsB-LacZ fusion protein, which contains a 10 aa linker between the Nterminal 132 aa of ChsB and LacZ, would be expressed under the control of the chsB promoter. In strain DL-4, it was predicted that the ChsD-LacZ fusion protein, which contains a 10 aa linker between the $\mathrm{N}$-terminal 126 aa of ChsD and LacZ, would be expressed under the control of the $c h s D$ promoter (see Methods). To avoid a position effect on expression, both $\mathrm{pB}-\mathrm{LAC}$ and $\mathrm{pD}$ LAC were integrated into the same $\arg B$ locus. The growth rates of strains BL-1 and DL-4 were almost the same as those of the relevant wild-type strains AU/A and $\mathrm{ABPU} / \mathrm{A}$, respectively, indicating that expression of the fusion proteins is not toxic (data not shown). We analysed the spatial expression pattern of these genes by staining $\beta$-galactosidase activity using $\mathrm{X}-\mathrm{Gal}$ as a substrate (Fig. 5). In strain BL-1, blue staining was observed in the hyphae (Fig. 5a), as well as in the conidiophore, especially in the metulae, phialides and conidia (Fig. 5b). A similar staining pattern was observed in strain DL-4 (Fig. 5c, d), while no staining was detected in the wild- type strains, AU/A and ABPU/A (data not shown). These expression data further support the proposition that ChsB and ChsD function not only in hyphal growth, but also in conidiation.

\section{DISCUSSION}

In this study, we found by using a conditional mutant of chsB that ChsB functions not only in hyphal growth but also in conidiation. By using double mutants of $c h s B$ and $c h s D$, we found evidence that the functional importance of ChsD increases in hyphal growth and in conidiation when the expression of $c h s B$ is repressed. The involvement of ChsB and ChsD in both hyphal growth and conidiation was supported by the expression analysis using lac $Z$ of E. coli as a reporter gene.

Our laboratory reported previously that a $c h s B$ disruptant could grow only as a heterokaryon containing both wild-type and disrupted chsB alleles (Yanai et al., 1994). The hyphae of a haploid chsB disruptant stopped growing immediately after the germination of conidia, with their hyphal tips swelling. Borgia et al. (1996) obtained viable homokaryons carrying the disrupted chs $B$ allele. Their mutants grew very slowly with a high degree of branching, and contained normal septa. The colonies did not form conidiophores and conidia, and became brown after about 4 days incubation. These phenotypes of the Borgia group's chsB disruptants are very similar to those of the repressed $c h s B$ conditional mutant generated in this study (Fig. 3, and our unpublished result). These results strongly suggest that $c h s B$ is necessary not only for hyphal growth but also for conidiation. The lethality of the $c h s B$ disruptant reported by Yanai et al. (1994) might be caused not only by the disruption of $c h s B$, but also by the production of the Cterminally truncated $\mathrm{ChsB}$, which could be expressed from the disrupted chsB locus. As shown in Fig. 4 and will also be mentioned below, the repression of $\operatorname{alc} A(\mathrm{p}):: \operatorname{chs} B$ is imperfect in nature, and the viability of the conditional chsB mutant BM-3/A1 may depend on this imperfection. While Borgia et al. (1996) reported that the chitin content of their chsB disruptant was almost the same as that of the parental strain, the 
chitin contents of the mutant BM-3/A1 in YGU and in YGU containing $0.6 \mathrm{M} \mathrm{KCl}$ were both approximately $70 \%$ of those of the wild-type strain (Table 5). It is possible that these differences were simply derived from the differences in the genetic backgrounds of the strains employed.

The results in Table 4 clearly show the defect of $c h s B$ mutants in conidiation, though the reduction in conidiation efficiency seems rather moderate. When we use repressible promoters for the analysis of the functions of gene products, we should pay attention to two points. First, when the repression of the transcription of the gene is leaky, effects milder than gene disruptions or deletions would be observed under the repressing condition. It has been reported that the expression from the alcA promoter is not completely repressed in minimal medium (Som \& Kolaparthi, 1994; McGoldrick et al., 1995). The results in Fig. 4 show that the chs $B$ transcripts are actually present even at $25 \mathrm{~h}$ after the shift from MMFT to MM plate. Second, when transcripts and/or products of the gene are stable after the shift to the repressive condition, the effects of transcriptional repression would be observed only after prolonged cultivation. As shown in Fig. 4 ( 5 h lane), this was also the case. Taking these precautions into account, it is possible that the contribution of $\mathrm{ChsB}$ to conidiation was underestimated in the carbon source exchange experiment (Table 4).

The defects in the hyphal growth of BM-3/A1 after the shift to a $c h s B$-repressing MM plate might have resulted in a reduction of the amount of conidiation-competent hyphae. However, in combination with the chsB expression in conidiophores and conidia (Fig. 5), we take our result to suggest that the function of ChsB is not limited to the generation of conidiation-competent hyphae but is directly involved in conidiation.

We noticed that the colony diameter of BM-3/A1 on MMFT plates is smaller than that of the wild-type strain. This finding might be caused by the dominant negative effect of the $c h s B$ overexpression. However, the chsB chsD double mutant DB-13 showed almost the same colony diameter as the wild-type strain. Therefore, it is likely that the $c h s B$ overexpression per se does not cause growth inhibition, but the simultaneous expression of $c h s D$ disturbs the fully wild-type growth.

A reduction in the growth rate of DB-13 compared to that of BM-3/A1 was observed on MM plates and YGU plates containing $0.6 \mathrm{M} \mathrm{KCl}$, but occurred only slightly on YGU plates (Table 3). This result indicates that, when $c h s B$ expression is repressed, the importance of the ChsD function in hyphal growth increases under conditions of higher osmolarity. ChsD seems to be dispensable for hyphal growth under conditions of low osmolarity in the absence of chs $B$ expression. It is possible that ChsD either does not function or its loss can be compensated for by other chitin synthases when the osmolarity of the culture media is low. However, the hyphal morphology of DB-13 was more disorganized than that of BM-3/A1 on YGU plates (Fig. 3g, h), suggesting that ChsD functions and contributes to hyphal morphology even under low-osmolarity conditions. To measure the growth rates on solid media, we inoculated many conidia at one point on a plate. It is possible that, as the colony diameter and hyphal density increase and glucose is consumed, leaky expression of chs $B$ occurs around the centre of a colony. Thus, it is likely that we have underestimated the effect of the $c h s D$ deletion on the growth rate on YGU. Supplementation of $0.6 \mathrm{M} \mathrm{KCl}$ did not greatly affect the ratio of the chitin content between DB-13 and BM-3/A1 (Table 5), suggesting that chitin content does not necessarily correlate with the degree of physiological disorder.

The conidiation efficiency of DB-13 was approximately $60 \%$ that of BM-3/A1 in the carbon source exchange experiment (Table 4). In this experiment, we used as media MMFT and MM, which have higher osmolarity than YGU. Since the deletion of $c h s D$ affected hyphal growth under these conditions (Table 3), the defect in conidiation may have been derived from some defects in hyphal growth. Furthermore, since we have obtained evidence that $\mathrm{ChsD}$ is present in conidiophore structures of the wild-type strain (Fig. 5), we propose that ChsD may also be directly involved in conidiation.

Our analyses of DB-13 revealed the importance of the $c h s D$ function under $c h s B$-repressing conditions. Beth Din et al. (1996) suggested that Chs4, the class IV chitin synthase of N. crassa, may serve as an auxiliary enzyme that supplements chitin synthesis when additional chitin is necessary. ChsD, also a class IV chitin synthase, may contribute to chitin synthesis in response to various conditions, including the chsB-repressing condition. Since the $c h s D$ deletion in the $c h s A$ mutants reduced the conidiation efficiency of the mutants (Motoyama et al., 1996; Culp et al., 2000), it is possible that the chs A deletion may also be the condition that increases ChsD activity. Alternatively, ChsD may have some redundant functions with ChsA and ChsB.

The phenotypes of the conditional chs $B$ mutants have some similarity to the phenotypes of the null mutants of the class III chitin synthase genes of other filamentous fungi, namely chs-1 of N. crassa and chsG of A. fumigatus. Further analysis of the chitin synthase functions using the conditional mutation in combination with mutations of other related genes will help to elucidate their coordination for fungal morphogenesis and differentiation, and to develop anti-fungal reagents effective against filamentous fungi.

\section{ACKNOWLEDGEMENTS}

This work was supported by a Grant-in-Aid for Scientific Research from the Ministry of Education, Science and Culture of Japan. M.I. was financially supported by research fellowships from the Japan Society for the Promotion of Science for Young Scientists. This work was performed using the facilities of the Biotechnology Research Center, The University of Tokyo. 


\section{REFERENCES}

Adams, T. H. \& Timberlake, W. E. (1990). Upstream elements repress premature expression of an Aspergillus developmental regulatory gene. Mol Cell Biol 10, 4912-4919.

Aguirre, J., Adams, T. H. \& Timberlake, W. E. (1990). Spacial control of developmental regulatory genes in Aspergillus nidulans. Exp Mycol 14, 290-293.

Aufauvre-Brown, A., Mellado, E., Gow, N. A. R. \& Holden, D. W. (1997). Aspergillus fumigatus chsE: a gene related to CHS3 of Saccharomyces cerevisiae and important for hyphal growth and conidiophore development but not pathogenicity. Fungal Genet Biol 21, 141-152.

Ballance, D. J. \& Turner, G. (1985). Development of a highfrequency transforming vector for Aspergillus nidulans. Gene 36, 321-331.

Beth Din, A. \& Yarden, O. (1994). The Neurospora crassa chs-2 gene encodes a non-essential chitin synthase. Microbiology 140, 2189-2197.

Beth Din, A. \& Yarden, O. (2000). The Neurospora crassa chs3 gene encodes an essential class I chitin synthase. Mycologia 92, 65-73.

Beth Din, A., Specht, C. A., Robbins, P. W. \& Yarden, O. (1996). chs-4, a class IV chitin synthase gene from Neurospora crassa. Mol Gen Genet 250, 214-222.

Borgia, P. T., lartchouk, N., Riggle, P. J., Winter, K. R., Koltin, Y. \& Bulawa, C. E. (1996). The chsB gene of Aspergillus nidulans is necessary for normal hyphal growth and development. Fungal Genet Biol 20, 193-203.

Bowen, A. R., Chen-Wu, J. L., Momany, M., Young, R., Szaniszlo, P. J. \& Robbins, P. W. (1992). Classification of fungal chitin synthases. Proc Natl Acad Sci US A 89, 519-523.

Casadaban, M. J., Martinez-Arias, A., Shapira, S. K. \& Chou, J. (1983). Beta-galactosidase gene fusions for analyzing gene expression in Escherichia coli and yeast. Methods Enzymol 100, 293-308.

Chen-Wu, J., Zwicher, J., Bowen, A. R. \& Robbins, P. W. (1992). Expression of chitin synthase genes during yeast and hyphal growth phases of Candida albicans. Mol Microbiol 6, 497-502.

Choi, W.-J., Santos, B., Durán, A. \& Cabib, E. (1994). Are yeast chitin synthases regulated at the transcriptional or the posttranslational level? Mol Cell Biol 14, 7685-7694.

Culp, D. W., Dodge, C. L., Miao, Y., Li, L., Sag-Ozkal, D. \& Borgia, P. T. (2000). The chs A gene from Aspergillus nidulans is necessary for maximal conidiation. FEMS Microbiol Lett 182, 349-353.

Fujiwara, M., Horiuchi, H., Ohta, A. \& Takagi, M. (1997). A novel fungal gene encoding chitin synthase with a myosin motor-like domain. Biochem Biophys Res Commun 236, 75-78.

Fujiwara, M., Ichinomiya, M., Motoyama, T., Horiuchi, H., Ohta, A. \& Takagi, M. (2000). Evidence that the Aspergillus nidulans class I and class II chitin synthase genes, $c h s C$ and $c h s A$, share critical roles in hyphal wall integrity and conidiophore development. J Biochem 127, 359-366.

Gold, S. E. \& Kronstad, J. W. (1994). Disruption of two genes for chitin synthase in the phytopathogenic fungus Ustilago maydis. Mol Microbiol 11, 897-902.

Horiuchi, H., Fujiwara, M., Yamashita, S., Ohta, A. \& Takagi, M. (1999). Proliferation of intrahyphal hyphae caused by disruption of $\operatorname{csm} A$ which encodes a class $\mathrm{V}$-chitin synthase with a myosin motor-like domain in Aspergillus nidulans. J Bacteriol 181, 3721-3729.

Kulmburg, P., Judewicz, N., Mathieu, M., Lenouvel, F., Sequeval,
D. \& Felenbok, B. (1992). Specific binding sites for the activator protein, ALCR, in the alcA promoter of the ethanol regulon of Aspergillus nidulans. J Biol Chem 267, 21146-21153.

May, G. (1992). Fungal technology. In Applied Molecular Genetics of Filamentous Fungi, pp. 1-27. Edited by J. R. Kinghorn \& G. Turner. London: Chapman \& Hall.

McGoldrick, C. A., Gruver, C. \& May, G. S. (1995). myoA of Aspergillus nidulans encodes an essential myosin I required for secretion and polarized growth. J Cell Biol 128, 577-587.

Mellado, E., Aufauvre-Brown, A., Gow, N. A. R. \& Holden, D. W. (1996). The Aspergillus fumigatus chs $C$ and chs $G$ genes encode Class III chitin synthases with different functions. Mol Microbiol 20, 667-679.

Miller, K. Y., Toennis, T. M., Adams, T. H. \& Miller, B. L. (1991). Isolation and transcriptional characterization of a morphological modifier: the Aspergillus nidulans Stunted (stuA) gene. Mol Gen Genet 227, 285-292.

Motoyama, T., Kojima, N., Horiuchi, H., Ohta, A. \& Takagi, M. (1994a). Isolation of a chitin synthase gene (chsC) of Aspergillus nidulans. Biosci Biotechnol Biochem 58, 2254-2257.

Motoyama, T., Sudoh, M., Horiuchi, H., Ohta, A. \& Takagi, M. (1994b). Isolation and characterization of two chitin synthase genes of Rhizopus oligosporus. Biosci Biotechnol Biochem 58, 1685-1693.

Motoyama, T., Fujiwara, M., Kojima, N., Horiuchi, H., Ohta, A. \& Takagi, M. (1996). The Aspergillus nidulans genes chs A and chsD encode chitin synthases which have redundant functions in conidia formation. Mol Gen Genet 251, 442-450. (Corrigendum in Mol Gen Genet 253, 520-528.)

Munro, C. A., Schofield, D. A., Gooday, G. W. \& Gow, N. A. R. (1998). Regulation of chitin synthesis during dimorphic growth of Candida albicans. Microbiology 144, 391-401.

Munro, C. A., Winter, K., Buchan, A., Henry, K., Becker, J. M., Brown, A. J., Bulawa, C.E. \& Gow, N. A. R. (2001). Chs1 of Candida albicans is an essential chitin synthase required for synthesis of the septum and for cell integrity. Mol Microbiol 39, 1414-1426.

Oakley, C. E., Weil, C. F., Kretz, P. L. \& Oakley, B. R. (1987). Cloning of the riboB locus of Aspergillus nidulans. Gene 53, 293-298.

Pammer, M., Briza, P., Ellinger, A., Schuster, T., Stucka, R., Feldmann, H. \& Breitenbach, M. (1992). DIT101 (CSD2, CAL1), a cell cycle-regulated yeast gene required for synthesis of chitin in cell walls and chitosan in spore walls. Yeast 8, 1089-1099.

Rowlands, R. T. \& Turner, G. (1973). Nuclear and extranuclear inheritance of oligomycin resistance in Aspergillus nidulans. Mol Gen Genet 126, 201-216.

Sambrook, J., Fritsch, E. F. \& Maniatis, T. (1989). Molecular Cloning: a Laboratory Manual, 2nd edn. Cold Spring Harbor, NY : Cold Spring Harbor Laboratory.

Shaw, J. A., Mol, P. C., Bowers, B., Silverman, S. J., Valdivieso, M. H., Durán, A. \& Cabib, E. (1991). The function of chitin synthase 2 and 3 in the Saccharomyces cerevisiae cell cycle. J Cell Biol 114, 111-123.

Som, T. \& Kolaparthi, V. S. R. (1994). Developmental decisions in Aspergillus nidulans are modulated by Ras activity. Mol Cell Biol 14, 5333-5348.

Specht, C. A., Liu, Y., Robbins, P. W. \& 8 other authors (1996). The chsD and chsE genes of Aspergillus nidulans and their roles in chitin synthesis. Fungal Genet Biol 20, 153-167.

Sudoh, M., Nagahashi, S., Doi, M., Ohta, A., Takagi, M. \& Arisawa, M. (1993). Cloning of the chitin synthase 3 gene from 
Candida albicans and its expression during yeast-hyphal transition. Mol Gen Genet 241, 351-358.

Sudoh, M., Yamazaki, T., Masubuchi, K., Taniguchi, M., Shimma, N., Arisawa, M. \& Yamada-Okabe, H. (2000). Identification of a novel inhibitor specific to the fungal chitin synthase. Inhibition of chitin synthase 1 arrests the cell growth, but inhibition of chitin synthase 1 and 2 is lethal in the pathogenic fungus Candida albicans. J Biol Chem 275, 32901-32905.

Valdivieso, M. H., Mol, P. C., Shaw, J. A., Cabib, E. \& Durán, A. (1991). CAL1, a gene required for activity of chitin synthase 3 in Saccharomyces cerevisiae. J Cell Biol 114, 101-109.

Wang, Z. \& Szaniszlo, P. J. (2000). WdCHS3, a gene that encodes a class III chitin synthase in Wangiella (Exophiala) dermatitidis, is expressed differentially under stress conditions. J Bacteriol 182, 874-881.

Xoconostle-Cázares, B., León-Ramirez, C. \& Ruiz-Herrera, J.
(1996). Two chitin synthase genes from Ustilago maydis. Microbiology 142, 377-387.

Xoconostle-Cázares, B., Specht, C. A., Robbins, P. W., Liu, Y., León, C. \& Ruiz-Herrera, J. (1997). Umchs5, a gene coding for a class IV chitin synthase in Ustilago maydis. Fungal Genet Biol 22, 199-208.

Yanai, K., Kojima, N., Takaya, N., Horiuchi, H., Ohta, A. \& Takagi, M. (1994). Isolation and characterization of two chitin synthase genes from Aspergillus nidulans. Biosci Biotechnol Biochem 58, 1828-1835.

Yarden, O. \& Yanofsky, C. (1991). Chitin synthase 1 plays a major role in cell wall biogenesis in Neurospora crassa. Genes Dev 5, 2420-2430.

Received 8 October 2001; revised 7 January 2002; accepted 10 January 2002. 